\title{
Characteristics of Hepatitis B Co-infection and Disease Evolution in HIV-Positive Paediatric Patients in Romania
}

\author{
Manuela Arbune ${ }^{1}$, Costinela Georgescu² \\ ${ }^{1}$ Department of Morphological Sciences, Dunarea de Jos University Faculty of Medicine and Pharmacy, Galati, Romania \\ ${ }^{2}$ Department of Pharmaceutical Sciences, Dunarea de Jos University Faculty of Medicine and Pharmacy, Galati, Romania
}

\begin{abstract}
Background: Infection with hepatitis B virus (HBV) contributes to morbidity and mortality in people living with human immunodeficiency virus (HIV).

Aims: The aim of the present study is to assess the influence of HBV co-infection in clinical characteristics and disease evolution among nosocomial HIV infected youth in Romania.

Study Design: Retrospective study.

Methods: We assessed HBsAg in 179 young people with nosocomial paediatric HIV infection. Demographic data, ALAT level, CD4-count, HIV-RNA, antiretroviral therapy and clinical behaviour were all statistically compared in patients who were HIV mono-infected and HBV-co-infected.

Results: The characteristics of patients are as follows: sex ratio M/F: 55.3\%, AIDS category $88 \%$, median nadir CD4-count 126/mm ${ }^{3}$. The prevalence of persistent $\mathrm{HBsAg}$ was $44.6 \%$. The mortality rate was $11.1 \%$, but no correlation with $\mathrm{HBsAg}$ was found. An average of three antiretroviral combinations is experienced by $97.7 \%$ of patients, including Lamivudine for over 5 years in $76 \%$ of cases and Tenofovir/Emtricitabine in $16.75 \%$ of patients. Patients under antiretroviral therapy achieved $53.07 \%$ sustained undetectable HIV-RNA and $40.78 \%$ restored immunity CD4-count $>500 / \mathrm{mm}^{3}$. ALAT enzyme was found to be high in $54.75 \%$ of patients.
\end{abstract}

Conclusion: During our research, we noticed that HBsAg was elevated in young people with HIV in Romania. Mortality rate was not statistically correlated to HBsAg. High ALAT levels are related with HBV, HDV co-infections, virological failure to antiretroviral treatment and the risk of death.

Key Words: HIV, Hepatitis B, antiviral agents, Romania

Received: 17.02 .2013

Accepted: 17.04.2013

\section{Introduction}

Human Immunodeficiency Virus (HIV) and Hepatitis B Virus (HBV) share similar transmission routes. The risk of chronic hepatitis $B$ is higher in co-infected HIV patients, while acute HBV mono-infection is self-limited in $90 \%$ of adults (1). Although efficient HBV vaccination is available, over two thirds of people with HIV have a serological marker of HBV infection (2). Across the European region, the prevalence of HBV varies, from low rates in Western countries $(<2 / 100,000)$ to high rates in Eastern countries $(>8 / 100,000)(3,4)$.

A high incidence of hepatitis $B$, especially in children, was reported in Romania twenty years ago. Due to continuation of a routine immunisation program for newborns - acting since 1995 - the incidence of hepatitis B decreased significantly between 1989 and 2004, from 43/100,000 to 8.5/100,000 (5). The peculiarity of HIV epidemics in Romania is represented by the predominance of a unique paediatric group of patients born between 1988 and 1990 that presented nosocomial infection during the first years of life. These children infected with HIV have grown into present-time adults with an active sex life fact that implies risks for spreading HIV and other sexually-transmitted diseases. The fact that many of these children survived for a long period of time could be related to antiretroviral therapy (ART) and to a prevalent HIV-F subtype (6). According to an epidemiological study of teenagers with HIV in Romania, $78 \%$ of HIV patients of this paediatric group had HBV markers of past or present infection and $43.4 \%$ had HBV replication markers (7).

The reciprocal impact of HIV and HBV requires a special approach towards the HIV-HBV co-infected population. Hepatitis B tends to be more severe in HIV-positive patients and increases the risk of mortality by liver diseases, as well as leading to the rapid progression of immunodeficiency (8). High HBV replication with consequent severe fibrosis is frequently related with HIV, while low or middle necroinflammation can be currently observed. HBV reactivation is more likely to occur in HIV patients even after HBs antibody response (1, 8). Hepatitis delta virus (HDV) represents a risk factor for the severity of chronic hepatitis $B$, but was not associated with progression to the stage of acquired immunodeficiency syndrome (AIDS) (9).

Most clinical trials have not clearly proven the role of HBV in HIV progression. However, there have been reports of higher risks of hepato-toxicity under ART and hepatic flares when ART is interrupted in HIV-HBV co-infected patients (10).

A group study on the meta-analysis of HBV infection regarding the mortality in HIV infected persons reported a 
decreased risk of death in post ART era (11). The benefits of ART by active drugs against both HIV and HBV have been proven by accurate HBV diagnostic and monitoring, including HBV-DNA level, serological tests, liver enzymes, ultrasound examination and $\alpha$-fetoprotein measurement (12). Lamivudine (LAM), as part of ART, has been widely experienced during the last years. Nowadays, this option is limited because of the risk of rapid emergence of LAM-resistant HBV $(13,14)$. Most current guidelines recommend ART for all HIV-HBV coinfected patients, regardless of the immunity level, including two active HBV drugs, such as Tenofovir (TDF) or Emtricitabine (EMC) (12). Interferon therapies are not conclusive regarding the effectiveness of HBV response in HIV patients; therefore, this treatment option is rare (15).

The aim of this study is to evaluate the impact of HBV coinfection on clinical characteristics and the evolution of nosocomial HIV infected youth in Romania.

\section{Material and Methods}

This research study retrospectively assessed 179 medical records of HIV patients in Romania followed up in the Infectious Diseases Clinic from Galati County. The inclusion criteria were: year of birth between 1988 and 1990, and HIV diagnosis before age 16 , prior to having an active sex life. The study also had a number of exclusion criteria: vertical HIV transmission and chronic hepatitis $\mathrm{C}$ co-infection. Demographic and behavioural data, HBs antigen ( $\mathrm{HBsAg})$, the history of antiretroviral therapy (ART), nadir of $\mathrm{CD}_{4}$-count, clinical and immunological HIV/AIDS stage by CDC-1993 criteria, were all collected in our database (January 2005) (16). We compared the characteristics of patients with positive and negative $\mathrm{HBsAg}$. Alanine Amino-transferase (ALAT) level, HBsAg, $\mathrm{CD}_{4}$-count, and HIV- ARN viral-load and ART drug combinations had been recorded every 6 months. The study endpoint was in December 2011 or the date of death. Values over 1.5 normal references (NV) were considered high ALAT values. Liver enzymes and HBsAg were tested by means of automatic immune-enzymatic assay in the Laboratory of Infectious Diseases Hospital from Galati. Persistent HBV infection was defined by positive HBsAg over six months, detected in two or more samples. During the follow-up of this study, we developed a $C D_{4}$ count technique by means of flow cytometer (FACS Calibur) and quantitative analysis of HIV-RNA viral load in blood by polymerase chain reaction (COBAS TaqMan HIV-1), both of which were performed in The Clinical Laboratories of National Institute for Infectious Diseases "Prof. Dr. Matei Balş" from Bucharest. The HIV-RNA undetectable level was considered below 400 copies/mL.

Updated ART was decided according to European and Romanian HIV therapeutic guidelines $(12,17)$. The ART levels that were studied were counted at the end of the study as the number of ART combinations and cumulative individual therapy period (month basis). We assessed the influence of common HIV and HBV antiviral drugs on HBsAg seroconversion, elevated ALAT levels and mortality rate, as the endpoint. The study was approved by The Ethical Committee of The
Infectious Diseases Clinic of "Dunarea de Jos" University from Galati.

Data was analysed by the XLSTAT statistical software. Mean or median values were calculated for continuous variables. The relationships between categorical variables were described with the cross-tabulation technique. Chi square test was used to examine the association between two categorical variables between groups. The results were processed as odds ratios $(\mathrm{OR})$ and $95 \%$ confidence intervals $(\mathrm{Cl})$, with statistical significance levels of $p<0.05$.

\section{Results}

Persistent positive HBsAg was found in 44.6\% (80/179) HIV patients from the paediatric cohort. Positive and negative HBsAg groups have similar distribution by sex and educational level. Most HBsAg positive patients are living in urban areas. Eighty-eight per cent of patients had documented clinical and/ or immunological AIDS criteria, but progression to AIDS was not significantly influenced by the persistent HBsAg (Table 1).

Global mortality rate was $11.1 \%(20 / 179)$, but the rate of death was not related to HBV co-infection $(p=0.654)$. The seroconversion rate of $\mathrm{HBsAg}$ was found to be $17.5 \%(14 / 80)$ in HIV-HBV co-infected patients. Although HBs seroconversion was not noticed in any deceased patients, the death rate was not statistically influenced $(p=0.169)$.

Evaluation of HDV markers was attained by $67 / 80$ patients with persistent HBsAg; $16 / 67$ were found to be positive. Coreplication of both HBV and HDV might enhance liver injury and significantly elevate ALAT, concordant with our findings (Table 2).

An average of three $(1,9)$ ART combinations were experienced by 179 of the patients until the end of 2011, including nucleoside/nucleotide class of common drugs for HIV and HBV, such as Lamivudine (LAM), Tenofovir (TDF) and Emtricitabine (EMC). Lamivudine was the most extensive experienced drug from the beginning of ART history while TDF and EMC became available for HIV treatment in Romania after 2010. In our study, $83 \%$ of patients were treated with LAM and $76 \%$ had over 5 years of LAM experience. Tenofovir and EMC were used together for most of the ART combinations and were supplied to $16.75 \%$ of patients. Replacing LAM with TDF/EMC was chosen for both HIV and HBV, according to the European AIDS Clinical Society algorithm of treatment in HIV/ HBV co-infection (12).

Both LAM and TDF/EMC have been administered in combinations that had at least two nucleoside reverse transcriptase inhibitors (NRTI) and a boosted protease inhibitor (IP/r) or non-nucleoside reverse transcriptase inhibitors (NNRTI). Some other drug classes were rarely combined, and this only happened in patients requiring salvage therapy.

Antiretroviral therapy evaluation at the end of this study emphasises that $53.07 \%$ of patients had sustained undetectable HIV-RNA and $40.78 \%$ had restored immunity with $C D_{4}-$ count $>500 / \mathrm{mm}^{3}$. Effectiveness of ART is independent of HBsAg. Elevated ALAT was recorded in $54.75 \%$ of patients, but only $28 \%$ scored three times over the normal value (NV). None 
Table 1. Baseline characteristics: a comparative study in HIV patients $\mathrm{HBs} A g+(N=80)$ vs. $\mathrm{HBs} A g-(N=99)$

\begin{tabular}{|c|c|c|c|c|}
\hline & HBsAg+ (\%) & HBsAg- (\%) & All (\%) & $p\left(X^{2}\right.$ Test $)$ \\
\hline Gender: Male & $58.75 \%$ & $52.52 \%$ & $55.30 \%$ & 0.404 \\
\hline Urban living aria & $67.5 \%$ & $46.46 \%$ & $55.86 \%$ & 0.004 \\
\hline Low educational level ( $\leq 4$ years) & $26.25 \%$ & $38.38 \%$ & $32.96 \%$ & 0.261 \\
\hline Alcohol users & $35 \%$ & $34.34 \%$ & $34.63 \%$ & 0.926 \\
\hline Smokers & $50 \%$ & $55.55 \%$ & $53.07 \%$ & 0.459 \\
\hline Drug users & $6.25 \%$ & $6.06 \%$ & 6.14 & 0.940 \\
\hline Clinic CDC-stage C & $62.5 \%$ & $67.67 \%$ & $65.37 \%$ & 0.469 \\
\hline Immunological stage 3 & $77.5 \%$ & $67.67 \%$ & $72.06 \%$ & 0.318 \\
\hline Mean of nadir CD4-count $/ \mathrm{mm}^{3}$ & 164,596 & 167,475 & 165,88 & - \\
\hline \multicolumn{5}{|c|}{$\begin{array}{l}\text { HIV: Human Immunodeficiency Virus; HBsAg+: Patients with positive HBs antigen; HBsAg-: Patients negative for HBs antigen; CDC- } \\
\text { stage: stage of HIV infection by Centers for Diseases Control classification; } \mathrm{p}\left(\mathrm{X}^{2} \mathrm{Test}\right): \mathrm{p} \text { significance value of Chi square Test was } \\
\text { considered below } 0.005\end{array}$} \\
\hline
\end{tabular}

Table 2. Statistical associations with elevated alanine-amino-transferase (ALAT)

\begin{tabular}{|c|c|c|c|c|}
\hline & 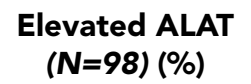 & $\begin{array}{l}\text { Normal ALAT } \\
(\mathbf{N}=\mathbf{8 1})(\%)\end{array}$ & $\mathrm{p}$ ( $\mathbf{X}^{2}$ Test) & $\begin{array}{l}\text { Odds ratio } \\
\text { (OR) }\end{array}$ \\
\hline Gender: Male & $62.24 \%$ & $46.91 \%$ & 0.040 & 1.866 \\
\hline Alcohol users & $40.81 \%$ & $27.16 \%$ & 0.050 & 1.841 \\
\hline Smokers & $57.14 \%$ & $48.14 \%$ & 0.230 & 1.435 \\
\hline Drug users & $8.16 \%$ & $3.84 \%$ & 0.223 & 2.281 \\
\hline Tuberculosis drugs & $42.85 \%$ & $38.27 \%$ & 0.534 & 1.201 \\
\hline LAM in previous treatment & $24.48 \%$ & $7.40 \%$ & 0.001 & 4.054 \\
\hline $\begin{array}{l}\text { HIV failure on ART } \\
\text { (detectable/undetectable VL) }\end{array}$ & $60.20 \%$ & $30.86 \%$ & $<0.001$ & 3.388 \\
\hline Restoration CD4>500/mm ${ }^{3}$ & $24.48 \%$ & $60.49 \%$ & $<0.001$ & 4.721 \\
\hline HBs Ag positive & $38.77 \%$ & $75.30 \%$ & $<0.001$ & 4.815 \\
\hline HDV-HBV co-infection* & $17.44 \%$ & $1.23 \%$ & $<0.001$ & 16.69 \\
\hline
\end{tabular}

of the 19 available NRTI, NNRTI and PI agents used for ART combinations were related to significant liver toxicity. It was also observed that ALAT levels increased more frequently in patients that stopped using LAM than in those who had ongoing LAM therapy $(p=0.001 ; O R=4.58)$. Increased ALAT can be correlated with HBsAg, HDV co-infection, virological HIV failure on ART and HIV-related mortality. However, high ALAT values are more commonly in patients with restored immunity of $\mathrm{CD}_{4}$-count over $500 / \mathrm{mm}^{3}$, which is probably explained by the pathology of immune reconstruction following ART (18). Increased serum ALAT levels are not statistically correlated with common risk factors such as alcohol, smoking, recreational drugs or concomitant anti-tuberculosis drugs for hepatotoxicity (Table 2).

\section{Discussion}

The high frequency of HIV/HBV co-infection in Galati is typical for Romanian paediatric HIV epidemics. Considering that HIV infection was transmitted in early childhood, diagnosis of the disease varied depending on the age of the patient, on other associated diseases and on the individual context. 
Taking into account the associated HIV-related depressed immunity and frequent immaturity of the immune system during early childhood, a high rate of HBV persistence is expected in spite of long periods without symptoms. Compared with EuroSIDA Cohort data (16.505 patients), the proportion of HIV/ HBV co-infection in our study is over six times higher: $44.6 \%$ vs. $7.1 \%$ (2). Testing methods for $\mathrm{HBsAg}$ and hepatitis $\mathrm{C}$ virus (HCV) antibodies are routine practices for a basic evaluation in newly diagnosed HIV patients $(12,17)$. Other investigations recommended by current guidelines for the management of HIV/HBV co-infection, such as HBV-DNA, non-invasive histological tests or other HBV and HDV serological markers, are not made available by the Romanian health insurance system in our clinic, thus our patients can rarely have them done. Assessment of HBV co-infection among HIV patients by HBsAg is not accurate enough for severe immunosuppressed conditions (1).

Co-replication of both HBV and HDV might enhance liver injury and significantly elevate ALAT, concordant with our findings. The frequency of HIV/HBV/HVD co-infection among young people in Romania is 1.64 times higher than EuroSIDA Cohort data: $23.8 \%$ vs. $14.5 \%$ (2). The assessment of HDV influence on mortality rate is not feasible because our deceased patients were not previously tested for this virus. Mortality is independent of HBV in our study, but the EuroSIDA study has reported a six times higher mortality rate related to HBV (2). This discrepancy can be explained by age differences between Romanian patients and those in the EuroSIDA Cohort report and also by the variable virulence of HIV and HBV subtypes present in European countries.

Long-term HIV infection in young people in Romania in the paediatric cohort shows the HIV/AIDS history from the last 23 years. Survival is the most important achievement of ART, although history recalls many errors that were made at the beginning of HIV therapy. The primary aim of ART combination, including LAM, was to suppress HIV replication and not necessarily HBV. The apparent influence of LAM on HBV was observed in $17.5 \%$ of HIV/HBV co-infected patients with HBs seroconversion. However, HIV resistance mutations can have a quite fast response, especially in non-adherent patients. Moreover, LAM benefits in the prevention and limitation of liver injuries depend on time response (15). Using LAM in patients with previous multiple therapies with resistant strains is a treatment strategy to maintain both HIV and HBV "viral fitness" when there are no other treatment options. Although current international guidelines recommend the use of TDF and $E M C$ in the first line of ART, they were only recently made available for our patients and are usually used in salvage therapy. Lamivudine was changed with TDF/EMC in thirty of our patients with ART failure and apparent HBV resistance (17). The testing procedures for HBV resistance in LAM therapy was not available for our patients. The assumption of Lamivudine resistance was based on long-term exposure to LAM and high ALAT levels. Normalisation of serum ALAT levels, without significant kidney toxicity, succeeded after six months of TDF/ EMC therapy. Future screening of TDF/ EMC patients as part of ART should be carefully taken into account for both HBV and HIV response. Discontinuity of TDF/EMC and HBV drug- resistance are related to hepatitis flare ups. The present peculiar aspects of HBV co-infection among Romanian youths call for a review of the therapeutic strategy. Up to date investigations for viral hepatitis diagnostic, new antiviral agents and liver transplants are future expectations for the efficient management of HIV/HBV co-infection.

In conclusion, the prevalence of persistent HBV is $44.6 \%$ on youth in HIV paediatric cohort in Galati. Mortality rate in HIV patients is not influenced by HBV co-infection. Long-term treatment for HIV with Lamivudine overlaps with HBs seroconversion in $17.5 \%$ of HIV/HBV co-infected patients. Immunological and HIV-virological failure under ART, the interruption of Lamivudine, HBV and HVD co-infections are predictable factors for increased ALAT values. The accurate assessment of HBV infection is necessary in order to improve the management of HIV/HBV co-infected young people in Romania.

Ethics Committee Approval: Ethics committee approval was received from "Dunarea de Jos" University from Galati.

Informed Consent: Written informed consent was waived because of the retrospective nature of the analysis but all patients previously permitted to the use of their medical records for the purpose of any form of clinical study.

Peer-review: Externally peer-reviewed.

Author contributions: Concept - M.A.; Design - M.A.; Supervision C.G.; Resource - M.A.; Materials - M.A.; Data Collection\&/or Processing M.A., C.G.; Analysis\&/or Interpretation - M.A., C.G.; Literature Search - M.A., C.G.; Writing - M.A., C.G.; Critical Reviews - C.G.

Conflict of Interest: No conflict of interest was declared by the authors.

Financial Disclosure: No financial disclosure was declared by the authors.

\section{References}

1. Wang HS, Han SH. Management of hepatitis B in special population. Clinical Liver Disease 2010;14:505-20. [CrossRef]

2. Konopnicki D, Mocroft A, de Wit S, Antunes F, Ledergerber B, Katlama C, et al. Hepatitis B and HIV: prevalence, AIDS progression, response to highly active antiretroviral therapy and increased mortality in the EuroSIDA cohort. AIDS 2005;19: 593-601. [CrossRef]

3. Liaw YF, Brunetto MR, Hadziyannis S. The natural history of chronic HBV infection and geographical differences. Antiviral Therapy 2010;15:25-33. [CrossRef]

4. Tekin Koruk S, Koruk I, Gursoy B, Çalisir C, Kuksell F, Yildiz Zeyrek F, et al. Hepatitis B and Hepatitis C Seroprevalence in the Center of Sanliurfa Province From Southeastern Anatolia Region and Related Risk Factors. Balkan Med J 2010;27:367-72.

5. Pitigoi D, Rafila A, Pistol A, Arama V, Molagic V, Streinu-Cercel A. Trends in hepatitis B incidence in Romania, 1989-2005. Euro Surveill 2008;13:pii=8012.

6. Apetrei C, Loussert-Ajaka I, Collin G, Letourneur F, Duca M, Saragosti $S$, et al. HIV type 1 subtype $F$ sequences in Romanian children and adults. AIDS Res Hum Retroviruses 1997;13:363-5. [CrossRef]

7. Ruta SM, Matusa RF, Sultana C, Manolescu L, Kozinetz CA, Kline $M W$, et al. High prevalence of hepatitis $B$ virus markers in romanian adolescents with human immunodeficiency virus infection. J Int AIDS Soc 2005;7:68. [CrossRef]

8. Audsley J, du Cros P, Goodman Z, McLean C, Mijch A, Lewin SR, et al. HIV replication is associated with increased severity of liver 
biopsy changes in HIV-HBV and HIV-HCV co-infection. J Med Virol 2012;84:993-1001. [CrossRef]

9. Soriano V, Grint D, d'Arminio Monforte A, Horban A, Leen C, Poveda $\mathrm{E}$, et al. Hepatitis delta in HIV-infected individuals in Europe. AIDS 2011;25:1987-92. [CrossRef]

10. Dore GJ, Soriano V, Rockstroh J, Kupfer B, Tedaldi E, Peters L, et al. Frequent hepatitis $B$ virus rebound among HIV-hepatitis $B$ virus-coinfected patients following antiretroviral therapy interruption. AIDS 2010;24:857-65. [CrossRef]

11. Nikolopoulos GK, Paraskevis D, Hatzitheodorou E, Moschidis Z, Sypsa V, Zavitsanos X, et al. Impact of Hepatitis B Virus Infection on the Progression of AIDS and Mortality in HIV-Infected Individuals: A Cohort Study and Meta-Analysis. Clin Infect Dis 2009;48:1763-71. [CrossRef]

12. European AIDS Clinical Society Guidelines for treatment of HIV infected adults in Europe, 2011. Version 6.0. Available online: http://www.europeanaidsclinicalsociety.org/images/stories/ EACS-Pdf/EACSGuidelines-v6.0-English.pdf.

13. Matthews GV, Avihingsanon A, Lewin SR, Amin J, Rerknimitr R, Petcharapirat $P$, et al. A randomized trial of combination hepatitis
B therapy in HIV/HBV coinfected antiretroviral naive individuals in Thailand. Hepatology 2008;48:1062-9. [CrossRef]

14. Ingiliz P, Valantin MA, Thibault V, Duvivier C, Dominguez S, Katlama $C$, et al. Efficacy and safety of adefovir dipivoxil plus pegylated interferon-alpha2a for the treatment of lamivudine-resistant hepatitis B virus infection in HIV-infected patients. Antivir Ther 2008;13:895-900.

15. Matthews GV, Manzini P, Hu Z, Khabo P, Maja P, Matchaba G, et al. Impact of lamivudine on HIV and hepatitis $B$ virus-related outcomes in $\mathrm{HIV} /$ hepatitis $B$ virus individuals in a randomized clinical trial of antiretroviral therapy in southern Africa. AIDS 2011;25:1727-35. [CrossRef]

16. 1993 revised classification system for HIV infection and expanded surveillance case definition for AIDS among adolescents and adults. MMWR Recomm Rep 1992;41:1-19.

17. Ministerul Sanatatii Publice. Ghidul TARV Romania 2011-2012. Available online: http://www.cnlas.ro/images/doc/ghidtarv.pdf.

18. Ofotokun I, Smithson SE, Lu C, Easley KA, Lennox JL. Liver enzymes elevation and immune reconstitution among treatmentnaïve HIV-infected patients instituting antiretroviral therapy. Am J Med Sci 2007;334:334-41.[CrossRef] 